\title{
Chronic kidney disease of unknown etiology in Sri Lanka
}

\author{
Senaka Rajapakse ${ }^{1}{ }^{\oplus}$, Mitrakrishnan Chrishan Shivanthan ${ }^{2}{ }^{\oplus}$, \\ Mathu Selvarajah ${ }^{3}$
}

${ }^{1}$ Faculty of Medicine, Department of Clinical Medicine, University of Colombo, Colombo, Sri Lanka, ${ }^{2}$ Vijaya Kumarathunga Memorial Hospital, Seeduwa, Sri Lanka, ${ }^{3}$ Nephrology Unit, General Hospital, Kurunegala, Sri Lanka

Introduction: In the last two decades, chronic kidney disease of unknown etiology (CKDu) has emerged as a significant contributor to the burden of chronic kidney disease (CKD) in rural Sri Lanka. It is characterized by the absence of identified causes for CKD. The prevalence of CKDu is 15.1-22.9\% in some Sri Lankan districts, and previous research has found an association with farming occupations.

Methods: A systematic literature review in Pubmed, Embase, Scopus, and Lilacs databases identified 46 eligible peer-reviewed articles and one conference abstract.

Results: Geographical mapping indicates a relationship between CKDu and agricultural irrigation water sources. Health mapping studies, human biological studies, and environment-based studies have explored possible causative agents. Most studies focused on likely causative agents related to agricultural practices, geographical distribution based on the prevalence and incidence of $\mathrm{CKDu}$, and contaminants identified in drinking water. Nonetheless, the link between agrochemicals or heavy metals and CKDu remains to be established. No definitive cause for CKDu has been identified.

Discussion: Evidence to date suggests that the disease is related to one or more environmental agents, however pinpointing a definite cause for CKDu is challenging. It is plausible that CKDu is multifactorial. No specific guidelines or recommendations exist for treatment of CKDu, and standard management protocols for CKD apply. Changes in agricultural practices, provision of safe drinking water, and occupational safety precautions are recommended by the World Health Organization.

Keywords: Sri Lanka, chronic kidney disease, CKDu, proteinuria, nephropathy, cadmium

\section{Introduction}

Sri Lanka has a population of approximately 20 million and agriculture is a major component of the economy. ${ }^{1}$ Chronic kidney disease (CKD) is a major burden on the healthcare system of Sri Lanka. Diabetes, hypertension, and the various forms of glomerulonephritis are well-recognized etiologies. ${ }^{2-4}$ With increasing prevalence of non-communicable diseases, in particular diabetes and hypertension, the burden of CKD is expected to rise. ${ }^{5}$ Since the $1990 \mathrm{~s}$, a new CKD, where no obvious cause is identifiable, has been described in Sri Lanka. This new condition has resulted in a rise in the incidence of CKD in rural Sri Lanka, and has been aptly named chronic kidney disease of unknown etiology (CKDu) ${ }^{6,7}$ Alternate suggested names for this condition include chronic agricultural nephropathy (CAN) and CKD of multifactorial origin (CKD-mfo). ${ }^{8,9}$ Similar entities with unknown cause for CKD exist in other parts

Correspondence to: Senaka Rajapakse, Faculty of Medicine, Department of Clinical Medicine, University of Colombo, 25, Kynsey Road, Colombo 08, Sri Lanka. Email: senaka@med.cmb.ac.lk of the world. Environmental agents and conditions, such as heavy metals and industrial chemicals, have been linked to the development of CKDu in other parts of the world..$^{10,11}$ In this systematic review, we seek to synthesize research on the cause(s) and treatment modalities of CKDu in Sri Lanka. We describe the epidemiology, clinical features, and diagnosis of this new entity in Sri Lanka, and explore the implicated etiologies.

\section{Methods}

Pubmed, Embase, Scopus, and Lilacs databases were searched in April of 2016 for publications containing at least one the following keywords: 'proteinuria,' 'chronic kidney disease,' 'CKD,' chronic renal failure,' 'CRF,' 'renal failure,' 'CKD-U,' 'CKDu,' 'renal,' 'kidney,' 'glomerular' AND 'Sri Lanka' OR 'Lanka' in any combination in the title, abstract or MeSH terms. The search was not time restricted. This search resulted in 727 papers. Endnote X5 ${ }^{\circledR}$ was used to combine, remove duplicates, and filter the abstracts. Two authors (SR and MCS) read 
the abstracts and identified relevant papers. All three authors read the full text of relevant papers, and relevant information was extracted and summarized in tables. The inter-rater agreement was $100 \%$. Related references were also included. Forty-six papers provided relevant information for our study objective. One peer-reviewed abstract of a presentation from an international scientific conference was also included in the review.

\section{Results \\ Defining CKDu}

CKD is considered to be of unknown origin in the absence of recognized conditions which cause renal disease, such as diabetes mellitus, chronic or severe hypertension, snake bite, glomerulonephritis or urological diseases, together with normal HbAlc $(<6.5 \%)$, blood pressure $<160 / 100 \mathrm{~mm} \mathrm{Hg}$ untreated or $<140 / 90 \mathrm{~mm} \mathrm{Hg}$ on up to two antihypertensive medications. This definition was developed by the Scientific Committee of the National Research Programme for CKDu launched by World Health Organization and the Ministry of Healthcare and Nutrition of Sri Lanka. ${ }^{12}$ The disease is graded into four stages based on proteinuria and cut-off values of eGFR (in $\mathrm{ml} /$ $\min / 1.73 \mathrm{~m}^{2}$ ): $>90,60-89,30-59,<30$. Stage one disease is characterized by proteinuria with preserved eGFR. ${ }^{13,14}$

\section{Epidemiology of CKDu}

A recent population-based cross-sectional study has estimated the point prevalence of CKDu between 15.1 and $22.9 \%$ in the Sri Lankan districts of Anuradhapura, Polonnaruwa, and Badulla, with an association with farming (OR 1.195, 95\% CI 1.007-1.418 $p=0.041$ ), in particular 'chena' cultivation ${ }^{13}$ ('chena' cultivation is a primitive form of cultivation where a farmer cuts down the trees of a small area of land in the jungle, sets fire to the woody growths, and cultivates the land, moving on the next year to fresh land). A community-based cross-sectional study conducted across five provinces in Sri Lanka showed that a previously established cause for CKD was not found in 84,81 , and $72 \%$ of CKD patients in the North Central Province, the Uva Province, and the Eastern Province, respectively. ${ }^{15}$ These three provinces are predominantly agricultural areas. In a study conducted in the Central and Southern Provinces where chena cultivation is uncommon, CKDu patients comprised 2.9 and $9.1 \%$ of the CKD burden, respectively. The point prevalence of CKD ranged from 2.3 to $9.5 \%$ in these areas. ${ }^{16}$ The burden of CKDu has been estimated at over $50 \%$ of all CKD cases in some tertiary nephrology centers in Sri Lanka close to affected areas. ${ }^{3}$ The incidence is low in Colombo, which is not an immediate catchment area for affected regions. ${ }^{4}$ The disease is more common in males between the ages of 40 and 69 years, in farming communities in rural Sri Lanka, with conflicting evidence of familial clustering., ${ }^{3,4,16-18}$ Studies using geographical mapping show that villages with a high prevalence of $\mathrm{CKDu}$ are often related to irrigation water sources and/or located below the level of the water table. ${ }^{19}$

\section{Clinical characteristics of CKDu}

Proteinuria (albumin-creatinine ratio $[A C R] \geq 30 \mathrm{mg} / \mathrm{g}$ ) and deranged renal function are the principal abnormalities seen in $\mathrm{CKDu}$. In the past, clinical presentation was often late, with many patients presenting in end-stage CKD. ${ }^{7}$ Since the diseases have gained wider recognition, community screening programs have been directed toward early identification of the condition. Clinically, CKDu is indistinguishable from CKD due to other causes. Edema and hypertension occur late in the disease progression. ${ }^{20}$ Increased excretion of alpha-1 microglobulin in the urine has been observed in $\mathrm{CKDu}$, and may be a potential early biomarker for screening purposes. ${ }^{16,17}$ However, other studies have demonstrated that alpha-1 microglobulin excretion is not exclusive to CKDu and is seen in a variety of renal tubular disorders as well as some non-nephrological conditions. ${ }^{21}$ Thus, there is inadequate evidence currently to recommend alpha-1 microglobulin as a screening test for CKDu in Sri Lanka.

\section{Histopathological findings in CKDu}

Tubulointerstitial disease is a prominent feature in subjects with suspected CKDu. ${ }^{15,16,20,22,23}$ A recent study in asymptomatic $\mathrm{CKDu}$ patients with proteinuria showed that the majority of subjects had varying degrees of interstitial fibrosis, interstitial inflammation, glomerulosclerosis, and tubular atrophy. ${ }^{18}$ In this study, interstitial fibrosis in more than $5 \%$ of cortical area was seen in $93 \%$ of study subjects. Severe renal scarring was uncommon. Tubular involvement appears to occur early in the disease, and this is further supported by the presence of elevated levels of urinary alpha-1 microglobulin (a tubular biomarker) in asymptomatic disease. ${ }^{17}$ The role of interstitial inflammation in the initiation of the disease is, however, unclear. In asymptomatic proteinuric patients, interstitial fibrosis without inflammation has been shown to be common. ${ }^{18}$ Nonetheless, it is postulated that interstitial inflammation may play a role in the progression of the disease. ${ }^{20,23}$ Another study conducted between 2008 and 2009 demonstrated glomerular changes, included global sclerosis (37.1\%), ischemic-type obsolescence, wrinkled and collapsed glomerular tufts (17.6\%), and glomerular enlargement (36\%). ${ }^{18}$ Focal segmental glomerulosclerosis was uncommon (3.6\%). Tubulointerstitial inflammation was universally present, and comprised mainly of mononuclear infiltrate. Vasculitic changes were notably absent. Overall, tubulointerstitial changes appear to predominate over glomerular and vascular changes in $\mathrm{CKDu}$. Hypertension appears to correlate with arteriolar hyalinosis $(p=0.02)$ and interstitial fibrosis $(p=0.06) .{ }^{18}$ A recently published study attempting to correlate clinical disease with the histopathological appearance from biopsy specimens showed progressive interstitial fibrosis 
and increasing inflammation corresponding with the stage of CKDu with tubular atrophy and glomerulosclerosis becoming apparent in stage 4 disease. ${ }^{24}$

\section{Causative factors - epidemiological studies}

Studies exploring the etiology of CKDu have focused on likely causative agents related to agricultural practices, geographical distribution based on the prevalence and incidence of $\mathrm{CKDu}$, and contaminants identified in drinking water. Health mapping studies, human biological studies, and environmental studies have explored a variety of possible causative agents. It is hypothesized from the distribution and epidemiology that the condition is linked to the environment and is likely to be related to human activities, in particular agriculture. Despite available data suggesting that $\mathrm{CKDu}$ is an environmentally acquired disease, no definitive causative factor has been identified. Geographic distribution and research findings favor a multifactorial etiology. ${ }^{25,26}$ Although heat stress has been proposed as a cause for CKDu in Central America, there is no evidence to attribute heat stress as a cause for the CKDu in Sri Lanka. ${ }^{9}$

Communities that source drinking water from shallow wells in close proximity to irrigation systems developed for agriculture with demonstrable seepage from irrigation system to the wells are more affected by CKDu compared to communities without irrigation seepage into wells. Affected villages are often located below the level of the water table. In contrast, communities living in geographical close proximity to irrigation systems who obtain drinking water from wells sourced by natural springs appear to be less affected by CKDu. ${ }^{19}$ Fertilizer run-off from upstream agricultural activities in the hill country and consequent changes in the ionicity causing a Hofmeistertype protein denaturing nephropathy has been postulated to be linked with $\mathrm{CKDu} .^{27}$

Studies have shown an association between drinking water drawn from wells and the use of pesticides with proteinuric nephropathy. ${ }^{14,28}$ The geographical distribution of the disease correlates with agricultural provinces. Thus, substances contained in drinking water, especially substances connected to agricultural practices, have been considered as possible causes of CKDu in many studies.

Various metals, metalloids, and minerals have been studied as likely etiological agents for CKDu. High levels of cadmium and fluoride, and complex interactions between the ratio of dissolved sodium and calcium in the presence of fluoride have been hypothesized to cause $\mathrm{CKDu}$ in endemic areas of Sri Lanka. ${ }^{29}$ Analysis of levels of different heavy metals in water, soil, plants, and fish from CKDu-prevalent regions initially suggested a likely exposure relationship between elevated cadmium levels in the environment and CKDu. Cadmium originates from the use of triple super phosphate fertilizers and weedicides. Water from several tributaries of the river Mahaweli, which is the primary source of irrigation in affected regions, has been shown to contain high levels of cadmium, well in excess of the recommended safe level of $5 \mathrm{ug} / \mathrm{L} \cdot{ }^{30-32}$ Hence cadmium has been extensively investigated as a putative causative agent in endemic areas; however studies have shown mixed findings. ${ }^{13,14,29-31,33-35}$ Two recent studies evaluating the role of heavy metal contaminants (arsenic [As], cadmium [Cd], lead $[\mathrm{Pb}]$, and uranium [U]) in drinking water in relation to urinary excretion of these agents concluded that CKDu cannot be clearly linked with the presence of these contaminants in drinking water. ${ }^{32,33}$ In another recent important study subjects from endemic regions had higher concentrations of excreted heavy metal and glyphosate in the urine, exceeding the reference range. ${ }^{36}$ The creatinine-adjusted values were also higher compared to non-endemic subjects. In this study, the researchers have proposed the synergistic effect of multiple heavy metals and agrochemicals as a possible mechanism for nephrotoxicity; however, further well-designed studies are needed to explore this concept further. Overall, none of the current epidemiological studies provide conclusive evidence of a causative link between heavy metals and CKDu.

Fluoride levels have also been found to be significantly elevated in endemic sources of water in affected regions. ${ }^{29,33}$ Ionic interaction between dissolved calcium, sodium, and fluoride triggered by a critical range in the ratio determined by the concentration of dissolved calcium and sodium has been studied as a putative cause of CKDu. ${ }^{29}$ The basis for this is that sources of drinking water in affected and non-affected regions have different ratios of calcium and sodium despite having similar levels of fluoride. The $\mathrm{Na}^{+} / \mathrm{Ca}^{++}$ratio in water has been observed to be in the range of 1.6 to 6.6 in CKDu regions as opposed to 34 to 469 for the non-endemic regions. When the $\mathrm{Na}^{+} / \mathrm{Ca}^{++}$ ratio is high, complexes of fluoride with $\mathrm{Na}^{+}$are created in drinking water, reducing both the toxicity of fluoride ions in the human body, and the absorption of $\mathrm{Ca}^{++}$. Conversely, higher $\mathrm{Ca}^{++}$levels which cause low $\mathrm{Na}^{+} / \mathrm{Ca}^{++}$ratios potentiates the damage on tubular cells in the presence of fluoride. It is notable however that the prevalence of CKDu is low in some areas with high fluoride levels, hence the ratios of $\mathrm{Ca}^{+}$and $\mathrm{Na}^{+}$ions probably determine its toxicity. ${ }^{29}$

\section{Causative factors - human studies}

High levels of urinary cadmium have been demonstrated in biological specimens from patients with CKDu compared to controls from affected and non-affected areas. ${ }^{13}$ This community-based cross-sectional study showed higher urinary cadmium levels in CKDu patients compared with healthy controls in both endemic and non-endemic areas; a dose responsive relationship between the CKDu stage and urinary cadmium concentration $(p<0.05)$ was seen. The mean urinary cadmium concentration was significantly elevated in patients with CKDu $(1.039 \mu \mathrm{g} / \mathrm{g})$ compared to controls from both endemic and non-endemic areas $(0.646 \mu \mathrm{g} / \mathrm{g}, p<0.001$ and $0.345 \mu \mathrm{g} / \mathrm{g}, p<0.05)$, 
respectively. This study also demonstrated that urinary lead and arsenic levels were similar in CKDu cases and healthy controls. Urine concentrations of sodium, potassium, calcium, magnesium, copper, zinc, and titanium in $\mathrm{CKDu}$ patients were within normal limits. The study concluded that cadmium exposure is a risk factor for the pathogenesis of $\mathrm{CKDu}$.

However in another study, higher levels of cadmium were found in the urine of healthy controls compared to cases with $\mathrm{CKDu}$, after correction for urinary creatinine levels. ${ }^{33}$ This finding disputes its causative relationship, and suggests that cadmium may merely be an incidental association. ${ }^{33}$ Another similar study from Japan also found lower levels of cadmium in the urine of patients with $\mathrm{CKDu}(n=106)$ compared to unaffected controls $(n=50)$, and cadmium levels were similar in affected Japanese patients with CKDu and unaffected controls from Sri Lanka. ${ }^{22}$ There was no significant dose-effect relationship between the concentration of arsenic, lead, or selenium in urine and the stage of $\mathrm{CKDu}$, however deficiency in selenium appeared to be an association. Serum and urinary studies do not suggest aluminum as a causative agent for CKDu. ${ }^{13,33}$ Tubular concentrating ability is impaired in $\mathrm{CKDu}$ and therefore metal concentration should be adjusted to urinary creatinine levels, and absolute urinary metal concentrations may be misleading. ${ }^{33}$

In a study where hair and urine samples collected from both CKDu patients and controls were analyzed for arsenic, $68 \%$ of CKDu patients and $28 \%$ of controls had urine arsenic levels above the threshold limit of $21 \mu \mathrm{g} / \mathrm{g}$ creatinine for arsenic-related nephropathy, thus suggesting a potential link between chronic arsenic toxicity and CKDu. ${ }^{37}$ A study comparing consumption of white rice as staple food in CKDu cases and matched controls showed no association with $\mathrm{CKDu} .{ }^{38}$

One study compared the levels of nephrotoxic mycotoxins in urine samples from patients with CKDu in the North-Central Province of Sri Lanka with healthy relatives and healthy Japanese subjects. ${ }^{39}$ Aflatoxins, ochratoxins, and fumonisins were detected in $61.29,93.5$, and $19.4 \%$, respectively, of the 31 patients studied. Though similar levels of these toxins were detected in healthy relatives of $\mathrm{CKDu}$ patients, the levels detected in Japanese subjects were much lower. Ochratoxin-A, a mycotoxin, has been purported as a likely agent, however levels in food items in $\mathrm{CKDu}$ endemic areas showed low levels, well below the maximum safe limits. ${ }^{40}$ The evidence supporting a causative role for nephrotoxic mycotoxins is insufficient at present.

Pesticides such as 2,4-D, 3,5,6-trichloropyridinol, p-nitrophenol, 1-naphthol, 2-naphthol, glyphosate, and AMPA have been detected in the urine of CKDu patients, however whether any of these agents appear to have a causative role in the etio-pathogenesis of CKDu has not been established. ${ }^{13} \mathrm{~A}$ causal relationship of $\mathrm{CKDu}$ to acetylcholine esterase (AChE) inhibitor pesticides was explored in a study conducted in affected areas when the disease entity was newly recognized..$^{41} \mathrm{AChE}$ levels were shown to be significantly lower in AChE inhibitor pesticide exposed-CKD patients as compared to unexposed-CKDu patients $(p<0.05)$, however a definite association could not be established in the study because AChE levels of exposed non-CKD farmers were historical data obtained from farmers living in another part of the country. Prior pesticide poisoning has not been linked to the development of CKDu. ${ }^{28}$ In a case control study, metabolites of neonicotinoid insecticides were shown to be lower in farmers with CKD compared to healthy controls living in Japan. ${ }^{42}$

Aristolochic acid is a well-known nephrotoxin. ${ }^{43}$ It's role in $\mathrm{CKDu}$ has not yet been evaluated adequately. In multivariate logistic regression analysis of a casecontrol study in 2007 at Anuradhapura Teaching Hospital, past use of ayurvedic/native medication was found to be significantly associated with $\mathrm{CKDu}(p<001) .{ }^{28}$ Some of these ayurvedic medications contain aristolochic acid. However, an increase in urothelial tumors which are seen in association with aristolochic acid are not reported in association with CKDu. Post-infective causes of CKDu have also been postulated, ${ }^{44}$ drawing parallels to the epidemic of CKD in Singapore three decades ago, however evidence is lacking. ${ }^{45}$

Despite various hypotheses being considered, no particular agent is conclusively established, including heavy metals, metalloids or minerals, mycotoxins, pesticides or native medications, to cause CKDu. Despite geographical associations with agricultural areas, no strong evidence exists linking any agriculture-related substance or practice with the development of CKDu. Thus, we feel it is premature to consider renaming it chronic agricultural nephropathy. ${ }^{9}$ It is quite plausible that $\mathrm{CKDu}$ is multifactorial and available evidence suggests that it is an environmentally acquired disease. ${ }^{8,11,26}$ Pinpointing a definite cause for $\mathrm{CKDu}$ is challenging task; the biggest difficulty is dissociating random associations from causative relationships.

\section{Disease progression \& death in CKDu}

In a cohort study carried out over 6.5 years $(n=143)$, hypertension was a consistent factor in disease progression throughout all stages of CKDu. Observational studies find that low body mass index is also associated with disease progression among patients with stage 2-3 $\mathrm{CKDu}$. Mortality rates increased with the stage of $\mathrm{CKDu}$, as expected. ${ }^{18}$

\section{Early detection or CKDu}

At present eGFR and urinary albumin-creatinine ratio are used for screening. Dipstick proteinuria appears unreliable and has low sensitivity. While some have suggested that urinary excretion of alpha 1-microglobulin measurements 
may have value as a screening test for detection of $\mathrm{CKDu}$, the evidence for this is weak. ${ }^{13,17,21}$

\section{Treatment of CKDu}

No specific guidelines or recommendations exist for treatment. Control of hypertension and prevention of further exposure to toxins are areas that may warrant further studies on the retardation of disease progression. General treatment strategies of CKD are meanwhile used in most settings.

\section{Discussion}

Current available scientific evidence is fragmented with gaps in knowledge about the etiology of Sri Lankan CKDu. Most available studies rely on suboptimal designs. Conflicting conclusions highlight the need for high quality, adequately powered studies to draw useful conclusions.

Enhancing nephrological services and infrastructure in affected areas, developing national level guidelines for renal replacement therapy of patients in combination with individual case management, fostering human resource development, promoting research, enhancing social services and training social workers to address problems faced by CKDu patients and their families, and ensuring streamlined resource management supported by adequate financing are key in combating this evolving catastrophe needing an urgent response. ${ }^{46}$

Phytoextraction and filtration of cadmium from water sources have been explored as possible preventive measures for $\mathrm{CKDu}$. However, since these are only postulated causative agents, it is questionable whether these measures are effective and economically feasible. ${ }^{47,48}$ Change in agricultural practices, provision of safe drinking water and occupational safety precautions are recommended by the WHO to reduce CKDu. ${ }^{49}$ The need for intensive early screening of patients in high-risk areas is obvious. Mapping of high-prevalence areas using geographical information systems is another potentially useful strategy to identify populations at risk of the condition. Regionaland national-level multipronged approaches targeting service provision, guidelines for renal replacement and case management, human resource development, research, social welfare, and financing are in different stages of development to tackle this evolving catastrophe.

Recent collaborative research into $\mathrm{CKDu}$ by the Sri Lanka Ministry of Health provoked a flurry of recommendations with regard to future directions of research and collaboration. ${ }^{50}$ In an attempt to encourage research into $\mathrm{CKDu}$, the University Grants Commission, which regulates the universities in the country, identified $\mathrm{CKDu}$ as a priority area for funding in their 2016 budget.

\section{Conclusions}

CKDu contributes significantly to the national burden of CKD in Sri Lanka, and the victims are often in their productive ages from the farming communities in rural Sri Lanka - mainly the North Central Region. Early disease is asymptomatic, and screening may help in detection and control of accelerating factors, e.g. hypertension; such strategies are common to all types of CKD, and no specific intervention is yet available for $\mathrm{CKDu}$. Symptomatic disease often presents late with advanced kidney disease. Despite a fair amount of research, the causative agent or agents remain an enigma, though it appears to be related to the environment and agriculture. Whether this is an entity with a single causative agent or a spectrum of nephropathies caused by different agents with overlapping clinic-pathological features with the same end point is currently unclear. Safe water for consumption and prudent farming practices may play a role in containing the epidemic.

\section{Author contributions}

MCS and SR conceptualized the review and carried out the literature survey. MCS and SR drafted the manuscript. MCS and SR critically reviewed the scientific content. All authors read and approved the final manuscript.

\section{Disclosure statement}

No potential conflict of interest was reported by the authors.

\section{ORCID}

Senaka Rajapakse (D) http://orcid.org/0000-0003-19656678

Mitrakrishnan Chrishan Shivanthan (D) http://orcid.org/ 0000-0003-4795-7232

MathuSelvarajah (DD http://orcid.org/0000-0002-2197-8927

\section{References}

1 Department of Agriculture SL. RICE Sri Lanka. Colombo: Department of Agriculture; 2006.

2 Rodrigo C, Samarakoon L, Rajapakse S, Lanerolle R, Sheriff R. Kidney disease in the elderly: a Sri Lankan perspective. Saudi J Kidney Dis Transpl. 2013;24:1285-90.

3 Athuraliya TN, Abeysekera DT, Amerasinghe PH, Kumarasiri PV, Dissanayake V. Prevalence of chronic kidney disease in two tertiary care hospitals: high proportion of cases with uncertain aetiology. Ceylon Med J. 2009;54:23-5.

4 Wijewickrama ES, Weerasinghe D, Sumathipala PS, Horadagoda C, Lanarolle RD, Sheriff RM. Epidemiology of chronic kidney disease in a Sri Lankan population: experience of a tertiary care center. Saudi J Kidney Dis Transpl. 2011;22:1289-93.

5 Abraham G, Varughese S, Thandavan T, Iyengar A, Fernando E, Naqvi SA, et al. Chronic kidney disease hotspots in developing countries in South Asia. Clini Kidney J. 2016;9:135-41.

6 Gooneratne IK, Ranaweera AK, Liyanarachchi NP, Gunawardane $\mathrm{N}$, Lanerolle RD. Epidemiology of chronic kidney disease in a Sri Lankan population. Int J Diab Dev Ctries. 2008;28:60-4.

7 Lanerolle RD, Nanayakkara S, Sheriffdeen AH, Sheriff R. Demographic characteristics of end stage renal disease in Sri Lanka. J Ceylon Coll Phy. 2000;33:2.

8 Wimalawansa SJ. Escalating chronic kidney diseases of multifactorial origin in Sri Lanka: causes, solutions, and recommendations. Environmental health and preventive medicine. 2014;19:375-94.

9 Jayasinghe S. Chronic kidney disease of unknown etiology should be renamed chronic agrochemical nephropathy. MEDICC Rev. 2014;16:72-4.

10 Karmaus W, Dimitrov P, Simeonov V, Tsolova S, Bonev A, Georgieva R. Metals and kidney markers in adult offspring of endemic 
nephropathy patients and controls: a two-year follow-up study. Environ Health. 2008; 7:1-11.

11 Soderland P, Lovekar S, Weiner DE, Brooks DR, Kaufman JS. Chronic kidney disease associated with environmental toxins and exposures. Adv Chronic Kidney Dis. 2010;17:254-64.

12 Epidemiology-Unit. Weekly epidimiological report Vol:36 No:49; research programme for chronic kidney disease of unknown aetiology in Sri Lanka. In: Palihawadana P, editors. Colombo: Ministry of Healthcare and Nutrition; 2009.

13 Jayatilake N, Mendis S, Maheepala P, Mehta FR. Chronic kidney disease of uncertain aetiology: prevalence and causative factors in a developing country. BMC Nephrol. 2013;14:180.

14 Wanigasuriya KP, Peiris-John RJ, Wickremasinghe R. Chronic kidney disease of unknown aetiology in Sri Lanka: is cadmium a likely cause? BMC Nephrol. 2011;12:32.

15 Athuraliya N, Abeysekera T, Karunaratne U, Chandrajith R, Kumarasiri R, Amerasinghe P, et al. Proteinuric-chronic kidney disease prevalence in rural communities of Sri Lanka: is uncertain aetiology a problem to be addressed? In: AaNZSo Nephrology, editor. 46th Annual Scientific Meeting of the Australian and New Zealand Society of Nephrology. Perth, Australia: Blackwell Publishing; 2010. p. 74 .

16 Athuraliya NT, Abeysekera TD, Amerasinghe PH, Kumarasiri R, Bandara P, Karunaratne U, et al. Uncertain etiologies of proteinuricchronic kidney disease in rural Sri Lanka. Kidney Int. 2011;80:121221.

17 Nanayakkara S, Komiya T, Ratnatunga N, Senevirathna ST, Harada KH, Hitomi T, et al. Tubulointerstitial damage as the major pathological lesion in endemic chronic kidney disease among farmer in North Central Province of Sri Lanka. Environ Health Prev Med. 2012; 17:213-21.

18 Senevirathna L, Abeysekera T, Nanayakkara S, Chandrajith R, Ratnatunga N, Harada KH, et al. Risk factors associated with disease progression and mortality in chronic kidney disease of uncertain etiology: a cohort study in Medawachchiya, Sri Lanka. Environ Health Prev Med. 2012;17:191-8.

19 Jayasekara JM, Dissanayake DM, Adhikari SB, Bandara P. Geographical distribution of chronic kidney disease of unknown origin in North Central Region of Sri Lanka. Ceylon Med J. 2013;58:6-10.

20 Selvarajah M, Weeratunga P, Sivayoganathan S, Rathnatunga N, Rajapakse S. Clinicopathological correlates of chronic kidney disease of unknown etiology in Sri Lanka. Indian J Nephrol. 2016; Epub ahead of print.

$21 \mathrm{Yu} \mathrm{H}$, Yanagisawa Y, Forbes MA, Cooper EH, Crockson RA, MacLennan IC. Alpha-1-microglobulin: an indicator protein for renal tubular function. J Clin Pathol. 1983;36:253-9.

22 Nanayakkara S, Senevirathna ST, Karunaratne U, Chandrajith R, Harada KH, Hitomi T, et al. Evidence of tubular damage in the very early stage of chronic kidney disease of uncertain etiology in the North Central Province of Sri Lanka: a cross-sectional study. Environ Health Prev Med. 2012;17:109-17.

23 Wijetunge S, Ratnatunga NV, Abeysekera DT, Wazil AW, Selvarajah $\mathrm{M}$, Ratnatunga CN. Retrospective analysis of renal histology in asymptomatic patients with probable chronic kidney disease of unknown aetiology in Sri Lanka. Ceylon Med J. 2013;58:142-7.

24 Wijetunge S, Ratnatunga NV, Abeysekera TD, Wazil AW, Selvarajah M. Endemic chronic kidney disease of unknown etiology in Sri Lanka: Correlation of pathology with clinical stages. Indian J Nephrol. 2015;25:274-80.

25 Noble A, Amerasinghe P, Manthrithilake H, Arasalingam S. Review of Literature on Chronic Kidney Disease of Unknown Etiology (CKDu) in Sri Lanka. Colombo: IWMI; 2014. p. 41.

26 Wanigasuriya $\mathrm{K}$. Update on uncertain etiology of chronic kidney disease in Sri Lanka's north-central dry zone. MEDICC Rev. 2014;16:61-5.

27 Dharma-Wardana MW, Amarasiri SL, Dharmawardene N, Panabokke CR. Chronic kidney disease of unknown aetiology and ground-water ionicity: study based on Sri Lanka. Environmental geochemistry and health. 2015;37:221-31.

28 Wanigasuriya KP, Peiris-John RJ, Wickremasinghe R, Hittarage A. Chronic renal failure in North Central Province of Sri Lanka: an environmentally induced disease. Trans R Soc Trop Med Hyg. 2007;101:1013-7.

29 Chandrajith R, Dissanayake CB, Ariyarathna T, Herath HM, Padmasiri JP. Dose-dependent $\mathrm{Na}$ and $\mathrm{Ca}$ in fluoride-rich drinking water-another major cause of chronic renal failure in tropical arid regions. Sci Total Environ. 2011;409:671-5.

30 Bandara JM, Wijewardena HV, Bandara YM, Jayasooriya RG, Rajapaksha H. Pollution of River Mahaweli and farmlands under irrigation by cadmium from agricultural inputs leading to a chronic renal failure epidemic among farmers in NCP, Sri Lanka. Environ Geochem Health. 2011;33:439-53.

31 Bandara JM, Wijewardena HV, Liyanege J, Upul MA. Chronic renal failure in Sri Lanka caused by elevated dietary cadmium: Trojan horse of the green revolution. Toxicol Lett. 2010;198:33-9.

32 Rango T, Jeuland M, Manthrithilake H, McCornick P. Nephrotoxic contaminants in drinking water and urine, and chronic kidney disease in rural Sri Lanka. Science Total Environ. 2015;518519:574-85

33 Nanayakkara S, Senevirathna S, Abeysekera T, Chandrajith R, Ratnatunga N, Gunarathne ED, et al. An integrative study of the genetic, social and environmental determinants of chronic kidney disease characterized by tubulointerstitial damages in the North Central Region of Sri Lanka. J Occup Health. 2014;56:28-38.

34 Wimalawansa SJ. The role of ions, heavy metals, fluoride, and agrochemicals: critical evaluation of potential aetiological factors of chronic kidney disease of multifactorial origin (CKDmfo/CKDu) and recommendations for its eradication. Environ Geochem Health 2016;38:639-78.

35 Bandara JM, Senevirathna DM, Dasanayake DM, Herath V, Abeysekara T, Rajapaksha KH. Chronic renal failure among farm families in cascade irrigation systems in Sri Lanka associated with elevated dietary cadmium levels in rice and freshwater fish (Tilapia). Environ Geochem Health. 2008;30:465-78.

36 Jayasumana C, Gunatilake S, Siribaddana S. Simultaneous exposure to multiple heavy metals and glyphosate may contribute to Sri Lankan agricultural nephropathy. BMC Nephrol. 2015;16:103.

37 Jayasumana MACS, Paranagama PA, Amarasinghe MD, Wijewardane KMRC, Dahanayake KS, Fonseka SI, et al. Possible link of Chronic arsenic toxicity with Chronic Kidney Disease of unknown etiology in Sri Lanka. J Nat Sci Res. 2013;3:64-73.

38 Siriwardhana EA, Perera PA, Sivakanesan R, Abeysekara T, Nugegoda DB, Weerakoon KG. Is the staple diet eaten in Medawachchiya, Sri Lanka, a predisposing factor in the development of chronic kidney disease of unknown etiology? - A comparison based on urinary beta2microglobulin measurements. BMC Nephrol. 2014;15:103.

39 Desalegn B, Nanayakkara S, Harada KH, Hitomi T, Chandrajith R, Karunaratne U, et al. Mycotoxin detection in urine samples from patients with chronic kidney disease of uncertain etiology in Sri Lanka. Bull Environ Contam Tox. 2011;87:6-10.

40 Wanigasuriya KP, Peiris H, Ileperuma N, Peiris-John RJ, Wickremasinghe R. Could ochratoxin A in food commodities be the cause of chronic kidney disease in Sri Lanka? Trans R Soc Trop Med Hygiene. 2008;102:726-8.

41 Peiris-John RJ, Wanigasuriya JK, Wickremasinghe AR, Dissanayake WP, Hittarage A. Exposure to acetylcholinesteraseinhibiting pesticides and chronic renal failure. Ceylon Med J. 2006;51:42-3.

42 Kabata R, Nanayakkara S, Senevirathna S, Harada KH, Chandrajith $\mathrm{R}$, Hitomi T, et al. Neonicotinoid concentrations in urine from chronic kidney disease patients in the North Central Region of Sri Lanka. J Occup Health. 2016;58:128-33.

43 LI X, Wang H. Aristolochic acid nephropathy: what we know and what we have to do. Nephrology. 2004;9:109-11.

44 Gamage CD, Sarathkumara YD. Chronic kidney disease of uncertain etiology in Sri Lanka: Are leptospirosis and Hantaviral infection likely causes? Med Hypotheses. 2016;91:16-9.

45 Woo KT, Choong HL, Tan HB, Chin YM, Chan CM. On uncertain etiologies of proteinuric-chronic kidney disease in rural Sri Lanka. Kidney Int. 2012;81:1277; author reply.

46 Wickremasinghe AR, Peiris-John RJ, Wanigasuriya KP. Chronic kidney disease of unknown aetiology in the North Central Province of Sri Lanka: trying to unravel the mystery. Ceylon Med J. 2011;56:1436.

47 Jayasinghe S. Chronic kidney disease in rural areas of Sri Lanka: an evolving catastrophe needing an urgent response. Ceylon Med J. 2014;59:94-6.

48 Bandara JM, Wijewardena HV, Seneviratne HM. Remediation of cadmium contaminated irrigation and drinking water: a large scale approach. Toxicol Lett. 2010;198:89-92.

49 Mendis S. Progress Report 13 Feb 2012: Chronic Kidney Disease of Uncertain Aetiology (CKDU) Sri Lanka. Colombo: World Health Organization; 2010.

50 Redmon JH, Elledge MF, Womack DS, Wickremashinghe R, Wanigasuriya KP, Peiris-John RJ, et al. Additional perspectives on chronic kidney disease of unknown aetiology (CKDu) in Sri Lankalessons learned from the WHO CKDu population prevalence study. BMC Nephrol. 2014; 15:125. 